\title{
Gobierno corporativo y deuda internacional de empresas latinoamericanas
}

\author{
Georgina Núñez Reyes, Ignacio Perrotini Hernández \\ y Francisco López-Herrera
}

\section{Resumen}

En este artículo se analiza la relación entre la emisión de deuda corporativa y un esquema de gobierno corporativo que incide en el desarrollo de los mercados financieros a través de la reducción de las asimetrías de información y los conflictos de interés. Para ello, analizamos el mercado de deuda internacional corporativa y evaluamos las normas sobre gobierno corporativo vigentes en el Brasil y en los cuatro países del Mercado Integrado Latinoamericano (MILA), a saber, Chile, Colombia, México y el Perú, por medio del indicador de la CEPAL, la CAF y el BID relativo a la emisión de deuda. Las pruebas recabadas mediante un modelo probit para datos de panel y un modelo pooled probit muestran que el cumplimiento de las normas sobre gobierno corporativo puede tener efectos favorables en la probabilidad de que una empresa logre emitir bonos internacionales en forma exitosa.

\section{Palabras clave}

Financiamiento de empresas, gobernabilidad corporativa, reglas y normas, mercados de capital, bonos, deuda de las empresas, modelos econométricos, América Latina y el Caribe

\section{Clasificación JEL}

F65, G15, G32

\section{Autores}

Georgina Núñez Reyes es Oficial de Asuntos Económicos de la División de Desarrollo Productivo y Empresarial de la Comisión Económica para América Latina y el Caribe (CEPAL). Correo electrónico: georgina.nunez@un.org.

Ignacio Perrotini Hernández es Coordinador de Posgrado de Economía en la Coordinación de Estudios de Posgrado de la Universidad Nacional Autónoma de México (UNAM). Correo electrónico: iph@unam.mx.

Francisco López-Herrera es Profesor e Investigador de la Facultad de Contaduría y Administración de la Universidad Nacional Autónoma de México (UNAM). Correo electrónico: francisco_lopez_herrera@yahoo.com.mx. 


\section{Introducción}

Gozzi y otros (2015) señalan que, en el período 1991-2013, la emisión de bonos corporativos en el mundo representó casi el $80 \%$ del capital total captado por las empresas y más del $90 \%$ del que estas negociaron en los mercados externos. Esto muestra la creciente importancia de esos mercados en la globalización financiera. Desde 2009 ha aumentado la influencia del mercado de los bonos corporativos en las decisiones de financiamiento de las empresas de América Latina. El monto total de la deuda internacional corporativa casi se ha cuadruplicado en relación con el período anterior a la crisis, como se puede observar en el gráfico 1. Dado que los instrumentos de deuda conllevan al pago de obligaciones futuras por parte de distintos agentes de la economía, lo que suceda en este mercado necesariamente tendrá repercusiones en la estabilidad de la economía global.

Gráfico 1

Deuda internacional corporativa total al final de cada trimestre, 2000-2015

(En miles de millones de dólares)

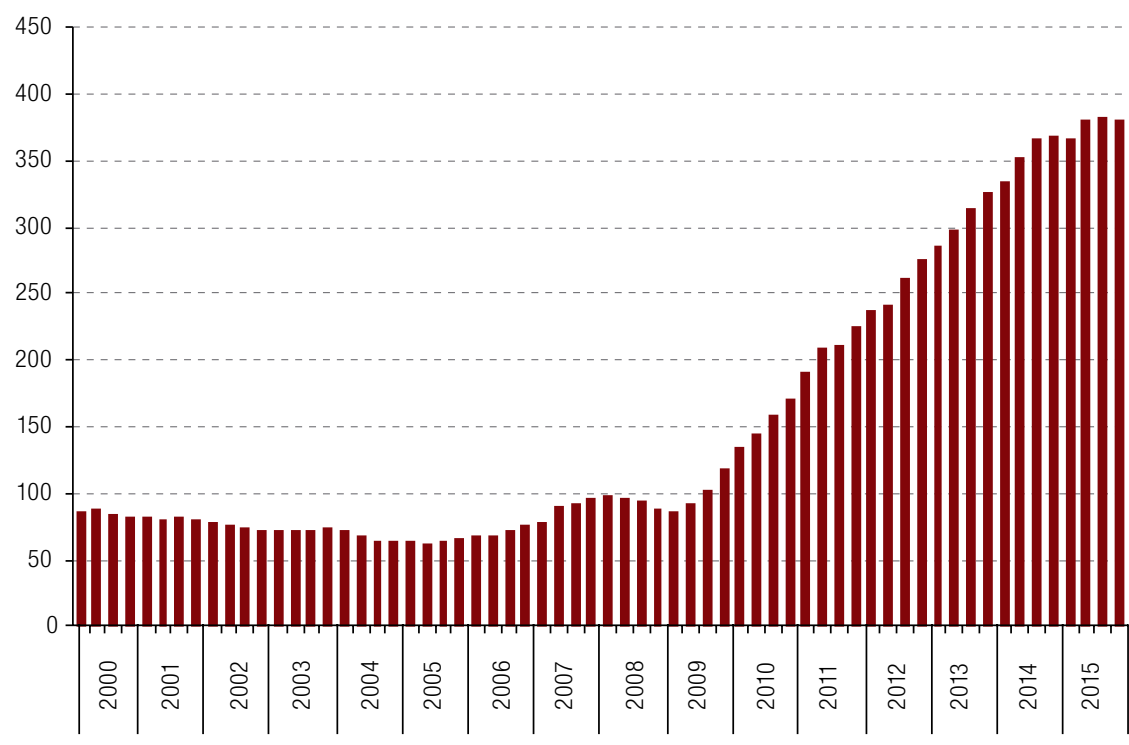

Fuente: Elaboración propia, sobre la base de Banco de Pagos Internacionales (BPI), "Debt securities statistics”, 2016 [en línea] https://www.bis.org/statistics/secstats.htm?m=6\%7C33\%7C615.

En los países en desarrollo, el impacto de las emisiones externas tiende a ser mayor que el de las internas. Çelik, Demirtas e Isaksson (2015) y Rodrigues, Kamil y Sutton (2015) atribuyen el crecimiento de la deuda corporativa en América Latina a las condiciones del mercado internacional, que se reflejan en la apreciación de las monedas de la región frente al dólar y en las tasas de interés cercanas a cero en los Estados Unidos. Hacia finales de 2014 hubo un cambio de escenario en los mercados financieros, cuando se dejó de aplicar la política estadounidense conocida como expansión cuantitativa (quantitative easing) ${ }^{1}$. Dicha política estaba en manos de la Reserva Federal y consistía en brindar estímulos monetarios a través de la compra de activos. Durante seis años, la expansión cuantitativa permitió trasladar liquidez (alrededor de 4,5 billones de dólares) a las economías en desarrollo y financiar con ello las inversiones corporativas.

Cuando finalizó la expansión cuantitativa, se trató de mantener el nivel de liquidez en los mercados financieros mediante la puesta en marcha de programas equivalentes implementados por

\footnotetext{
1 El objetivo de la expansión cuantitativa fue reducir las tasas de interés a largo plazo para estimular la demanda agregada y promover el crecimiento económico.
} 
los bancos centrales del Japón y Europa (2,5 billones de dólares). El conjunto de medidas adoptadas condujo a las principales economías desarrolladas a poner en práctica políticas monetarias expansivas (Núñez y Oneto, 2014)². Según Pérez Caldentey (2017), desde que se comenzaron a implementar las políticas de expansión cuantitativa, la participación de los mercados internacionales de bonos en el total del crédito mundial a los no residentes ha aumentado del $40 \%$ al $48 \%$. Los actores más activos y dinámicos fueron los residentes de Asia y América Latina. Entre 2009 y 2015, el volumen de las emisiones de deuda internacional de esas regiones se expandió un $12 \%$, mientras que en los países desarrollados se expandió un 0,23\% en el mismo período.

Este escenario de aumento de deuda, aunado a los escándalos corporativos surgidos durante la crisis de 2008, llevó a los compradores de bonos, especialmente en el mundo desarrollado, a ejercer una mayor presión para mejorar las prácticas de gobierno corporativo de las empresas emisoras $^{3}$. En particular en el caso del sistema financiero, el tema del gobierno corporativo como herramienta para mitigar el riesgo ha surgido con mucha fuerza como un medio de proteger a los inversionistas de posibles fraudes, mediante el incremento del flujo y la reducción de las asimetrías de información entre las partes involucradas en una transacción con emisiones de deuda, como en las fusiones o las adquisiciones.

El crecimiento del nivel de apalancamiento de las empresas, causado en parte por el aumento de la emisión de instrumentos de deuda que tuvo lugar desde 2010, se dirigió a la reestructuración de deuda ya existente (Gozzi y otros, 2015). Esto alertó a los mercados y a las autoridades regulatorias en cuanto a los posibles efectos sistémicos que el incumplimiento de los pagos podría tener sobre las economías nacionales, regionales y mundiales: la tasa de morosidad de los bonos mundiales ha alcanzado su nivel más alto desde 2009, según S\&P (Financial Times, 2016). En los procesos de integración como el del Mercado Integrado Latinoamericano (MILA) ${ }^{4}$, es preciso armonizar las regulaciones y las normas prevalecientes en cada país, de tal manera que el proceso integrador produzca el menor número de distorsiones en los mercados nacionales cuyos gobiernos corporativos tienen mejor desempeño. La heterogeneidad de las limitaciones que tienen las estructuras de gobierno corporativo para atender los desafíos que presenta el desarrollo y la integración de los mercados financieros, que incluyen las fallas de mercado (asimetría de información), junto con las limitaciones a las que se enfrentan los esquemas regulatorios y su aplicación, hacen necesario un análisis más detallado.

2 Según Rogoff (2015), con la expansión cuantitativa la Reserva Federal envió la señal de que, si las tasas de interés a largo plazo aumentaban bruscamente, el banco central estaba dispuesto a incurrir en grandes pérdidas, manteniendo una gran cantidad de bonos de interés bajo. En la medida en que el banco central tan solo comprara deuda pública, las pérdidas tenían poco significado económico. Que los bancos centrales rellenaran sus carteras con deuda a largo plazo de bajo rendimiento ayudó a convencer a los inversores de que mantendrían bajas las tasas de interés a corto plazo durante el mayor tiempo posible. Rogoff considera que la expansión cuantitativa es un instrumento débil e incierto comparado con la política normal de tasas de interés.

3 En un artículo publicado en América Economía se da cuenta de cómo, a partir de una serie de escándalos de algunas empresas de la región, se ponían en evidencia fallas en sus gobiernos corporativos. Dichas fallas se atribuían a deficiencias en los controles de los órganos decisorios que afectaron los fondos de pensiones y permitieron que se desviaran fondos hacia campañas y partidos políticos, además de que se violaran leyes electorales. Uno de estos casos fue el de la empresa SQM de Chile (Tromben y otros, 2015). Otros casos, como el de Petrobras, han sido aún más dramáticos, ya que han puesto en peligro la salud financiera de la empresa propiedad del Estado. En Chile, la propuesta de modificar la regulación apunta a desagregar más la información sobre los conflictos de interés, la administración de riesgos, las denuncias internas, las compensaciones y la participación en las juntas de accionistas, entre otras materias.

4 El Mercado Integrado Latinoamericano (MILA), actualmente conformado por las bolsas de valores de Chile, Colombia, México y el Perú, inició operaciones en noviembre de 2010. Originalmente, el mercado estaba integrado por el Perú, Colombia y Chile, y contaba con 563 emisoras. En términos de capitalización bursátil sumaba 660.985 millones de dólares: Chile representaba el 50,6\% (334.461 millones), Colombia, el 33,55\% (221.775 millones), y el Perú, el 15,85\% (104.749 millones). Esto convertía al MILA en el segundo mercado con mayor capitalización bursátil después del Brasil, que tenía 1.747.315 millones de dólares. Respecto al volumen de negocios, el MILA se constituyó en el tercer mercado de América Latina, con 57.000 millones de dólares al año, después del Brasil (633.000 millones) y de México (87.000 millones). México, cuya capitalización bursátil era de 457.997 millones de dólares, se integró al MILA en 2014. En el Perú, la mayor oferta de títulos provenía de las mineras (53\%), en Colombia, de la industria (78\%) y, en Chile, de los servicios de la capitalización (32\%). Véanse más detalles en [en línea] mercadomila.com. 
Un gobierno corporativo eficiente, inserto en un marco legal amplio y en una estrategia empresarial, contribuye al desarrollo de los mercados de capitales, reduce las fallas de mercado y facilita el acceso a fuentes diversas de financiamiento. El objetivo de este artículo es aportar pruebas sobre cómo se incrementan las posibilidades de colocar deuda de forma exitosa gracias a la existencia de un gobierno corporativo eficiente.

La estructura de este artículo es la siguiente: a continuación presentamos algunos antecedentes del mercado de deuda latinoamericano y de los bonos internacionales como la forma preferida que asume el endeudamiento corporativo a partir de la crisis financiera de 2008; el análisis hace especial énfasis en el Brasil y en los cuatro países integrantes del MILA; después, discutimos el concepto de gobierno corporativo con base en los marcos normativos y los códigos de buenas prácticas que hay sobre el tema en cada uno de los países seleccionados; posteriormente, presentamos un análisis econométrico para explicar la influencia de una serie de variables relevantes, incluida la relacionada con el gobierno corporativo, en la emisión de deuda, y, por último, presentamos las conclusiones.

\section{El mercado de deuda latinoamericano}

En el período que va del 1 de enero de 2005 al 31 de diciembre de 2015 se observa un enorme dinamismo en los mercados de deuda, particularmente en los de deuda corporativa. El total de las emisiones internacionales corporativas de 23 países de América Latina y el Caribe ${ }^{5}$ durante el período 2005-2015 alcanzó 1,36 billones de dólares, que corresponden a 14.630 emisiones internacionales y nacionales. Del total emitido, cerca de un 60\% (805.000 millones de dólares) es deuda internacional. El Brasil, con casi el 40\%, y México, con el 29\%, fueron los principales emisores de deuda corporativa internacional (véase el cuadro 1). En términos del número de emisiones, el Brasil representa casi el $75 \%$ del total, seguido por Chile, con cerca del 10\%.

Cuadro 1

América Latina y el Caribe: emisiones de deuda internacional de las empresas, 2005-2015

\begin{tabular}{lcccc}
\hline & $\begin{array}{c}\text { Valor } \\
\text { (en miles de millones } \\
\text { de dólares) }\end{array}$ & $\begin{array}{c}\text { Valor } \\
\text { (en porcentajes) }\end{array}$ & $\begin{array}{c}\text { Número } \\
\text { (en unidades) }\end{array}$ & $\begin{array}{c}\text { Número } \\
\text { (en porcentajes) }\end{array}$ \\
\hline Total de emisiones & 805,25 & & 6728 & 74,8 \\
\hline Brasil & 320,89 & 39,9 & 5032 & 6,0 \\
\hline México & 236,88 & 29,4 & 403 & 9,9 \\
\hline Chile & 72,88 & 9,1 & 666 & 0,5 \\
\hline Venezuela (República Bolivariana de) & 50,64 & 6,3 & 34 & 1,1 \\
\hline Colombia & 34,81 & 4,3 & 73 & 1,5 \\
\hline Perú & 32,71 & 4,1 & 102 & 1,9 \\
\hline Argentina & 15,71 & 2,0 & 130 & 4,3 \\
\hline Otros & 40,73 & 5,1 & 288 & \\
\hline
\end{tabular}

Fuente: Elaboración propia, sobre la base de información de Bloomberg.

Las emisiones internacionales consideradas en la muestra se realizaron originalmente en 22 monedas. En el gráfico 2 se observa que el $88 \%$ se realizaron en dólares, seguidas de lejos por las emisiones en euros (7\%). Este predominio incluso pudo haberse acentuado en el período más reciente, debido al fortalecimiento del dólar desde finales de 2014.

En el gráfico 3 se muestra la distribución de la deuda por sector de la economía respecto al monto total emitido. Según cifras de Bloomberg, las emisiones de mayor valor se concentran en el

\footnotetext{
5 Argentina, Brasil, Chile, Colombia, México, Perú, Estado Plurinacional de Bolivia, Ecuador, Paraguay, Uruguay, República Bolivariana de Venezuela, Belice, Costa Rica, El Salvador, Guatemala, Nicaragua, Panamá, Bahamas, República Dominicana, Jamaica, San Vicente y Las Granadinas, Barbados, y Trinidad y Tabago.
} 
sector de la energía, que representa el 29\% del valor y tan solo el 3,2\% del número total de emisiones de bonos de la región. Por otra parte, el sector financiero es responsable del $28 \%$ del monto total emitido y de casi el $83 \%$ del número total de emisiones de deuda internacional.

\section{Gráfico 2}

América Latina y el Caribe: denominación de las emisiones de deuda internacional, 2005-2015 (En porcentajes)
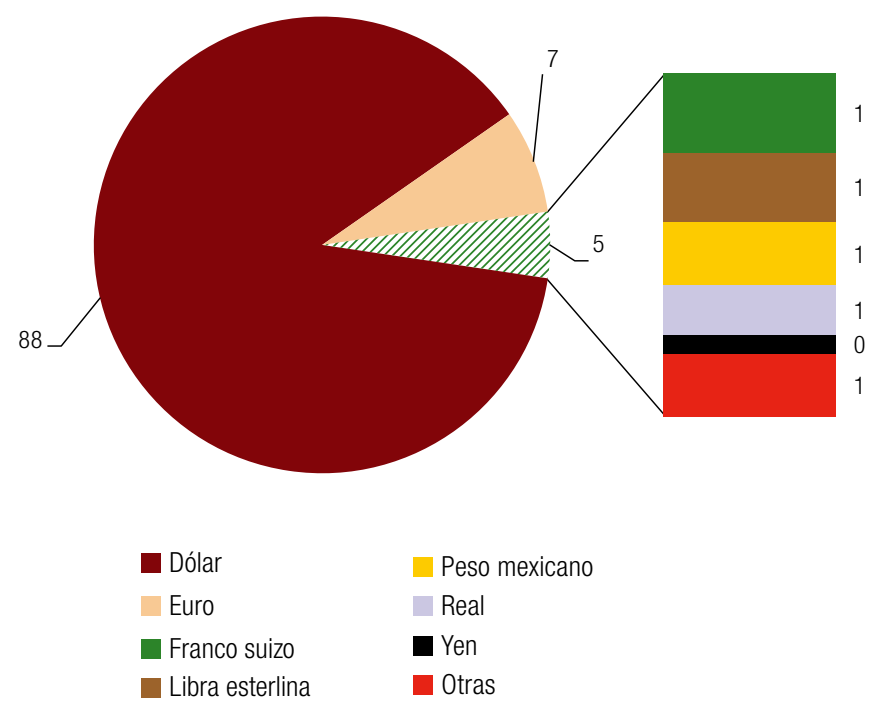

Fuente: Elaboración propia, sobre la base de información de Bloomberg.

\section{Gráfico 3}

América Latina y el Caribe: distribución del valor de las emisiones de deuda internacional por sector de actividad, 2005-2015

(En porcentajes)

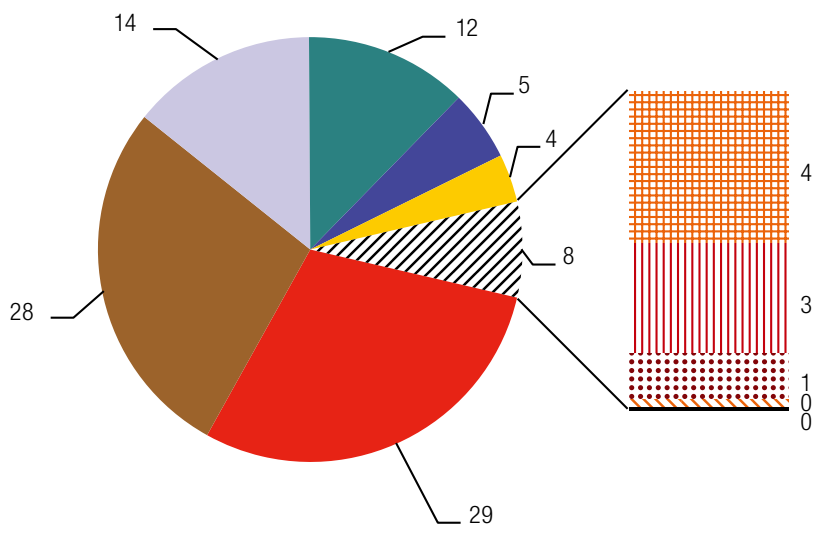

$\begin{array}{lll}\text { Energía } & \text { ::: Gobierno } & \text { Productos industriales } \\ \text { Materiales } & \text { Tecnología } & \text { III Productos de consumo discrecional } \\ \text { Productos básicos de consumo } & \text { Sector financiero } & \text { NN Cuidados de la salud } \\ \text { \# Servicios públicos } & \text { Comunicaciones } & \end{array}$

Fuente: Elaboración propia, sobre la base de información de Bloomberg.

Tan solo siete países de la región realizaron el 95\% de las emisiones: los cuatro del MILA, la Argentina, el Brasil y la República Bolivariana de Venezuela. Los 16 países restantes son responsables, en conjunto, de tan solo el $5 \%$ de la emisión internacional corporativa de la región. El porcentaje mayor de emisiones proviene del sector no financiero (véase el gráfico 4). 
Gráfico 4

América Latina y el Caribe (22 países): emisiones de deuda del sector financiero y del no financiero, 2005-2015

(En porcentajes)

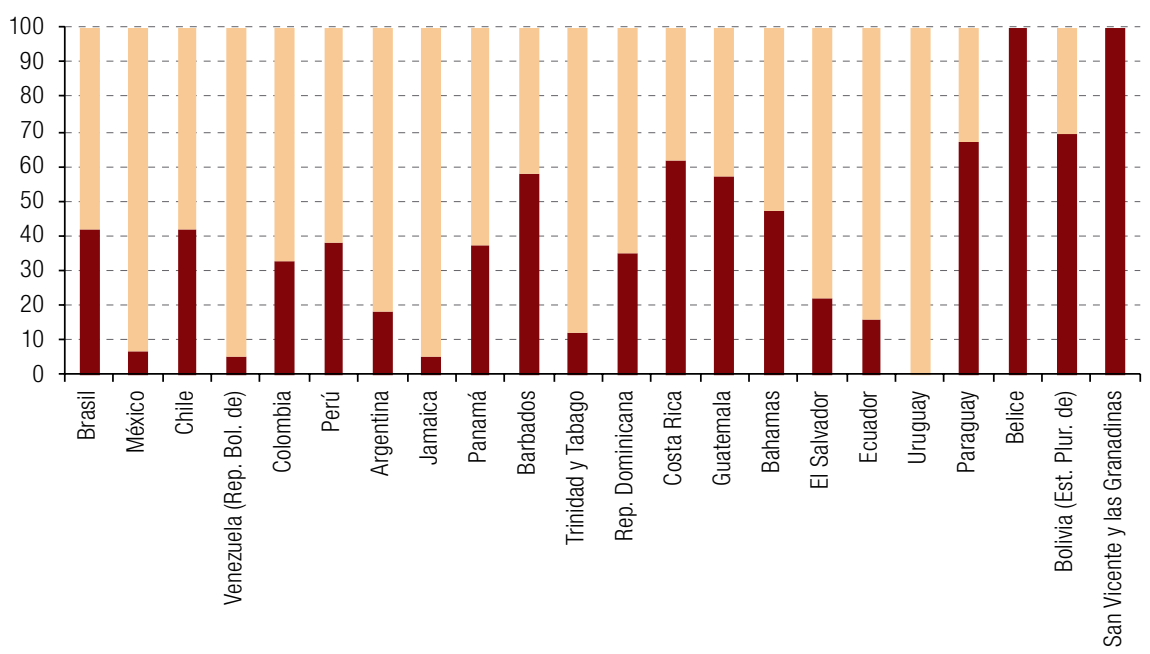

Sector financiero Sector no financiero

Fuente: Elaboración propia, sobre la base de información de Bloomberg.

En el gráfico 5 se muestran los países donde se colocó la deuda corporativa. El 26\% de la deuda total se radicó en el mercado mexicano y el 19\% en el Brasil. Si bien el 10\% de la deuda total se colocó a través de una empresa en los Países Bajos, el riesgo de esa emisión se encuentra en algún país de América Latina y el Caribe. Por otra parte, en las Islas Caimán, territorio recurrentemente considerado paraíso fiscal, se concentra el 9\% de la deuda total colocada.

\section{Gráfico 5}

Países donde se colocó la deuda corporativa de América Latina y el Caribe, 2005-2015

(En porcentajes)

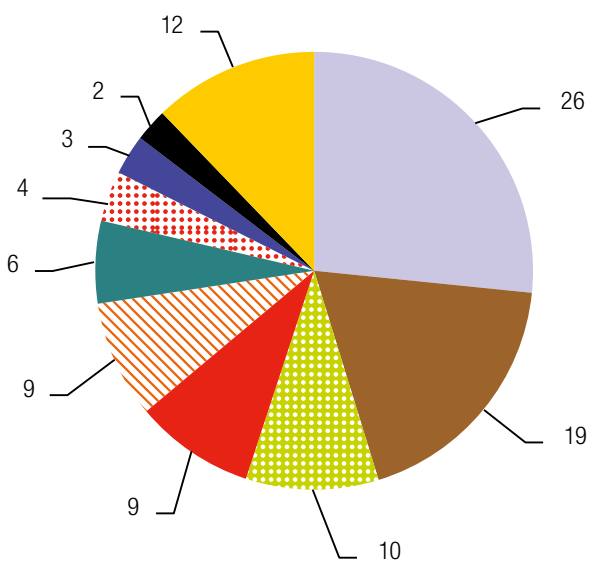

$\begin{array}{lll}\text { México } & \text { Brasil } & \text { Países Bajos } \\ \square \text { Islas Caimán } & \mathbb{N} \text { Chile } & \text { Venezuela (Rep. Bol. de) } \\ \text { :i: Estados Unidos } & \square \text { Colombia } & \mathbf{\square} \text { Perú } \\ \text { Otros } & & \end{array}$

Fuente: Elaboración propia, sobre la base de información de Bloomberg. 


\section{Gobierno corporativo, marcos normativos y códigos de buenas prácticas}

La inexistencia de mecanismos autónomos que permitan monitorear de forma eficiente e integral el nivel de riesgo de la empresa es una de las principales deficiencias que se pusieron de manifiesto durante los años recientes. La falta de una instancia especializada en la estructura del gobierno corporativo se puede percibir en el nivel de la toma de decisiones de la empresa ${ }^{6}$.

En el caso de los países del MILA, los criterios de calificación del riesgo de las emisiones corporativas son similares a los que utilizan las calificadoras de riesgo y con ellos se evalúa la capacidad del emisor para cumplir con los pagos comprometidos a los inversores dentro de los términos y plazos preestablecidos en los contratos. Sin embargo, al analizar las cifras surgen algunas dudas respecto de cómo se controla el uso y la efectividad de algunos métodos de evaluación del riesgo y el proceso de toma de decisiones de la empresa en materia de apalancamiento ${ }^{7}$. Los marcos normativos que rigen a los gobiernos corporativos y que se analizan en esta sección estuvieron vigentes durante el período 2005-2015. Las decisiones de las emisoras se ajustan a una estructura de gobierno corporativo efectiva percibida y valorada por los distintos agentes del mercado, como los inversionistas institucionales, las calificadoras de crédito y los bancos colocadores de títulos de deuda. Con el índice de gobierno corporativo que construimos aquí tratamos de determinar si existe correspondencia entre estos rasgos del gobierno corporativo (sujeto a la normativa vigente y a los códigos de buenas prácticas) y el mayor acceso, en mejores condiciones, al mercado de bonos.

Para medir el conjunto de normas de los gobiernos corporativos se utiliza el indicador elaborado por la Comisión Económica para América Latina y el Caribe (CEPAL), el Banco de Desarrollo de América Latina (CAF) y el Banco Interamericano de Desarrollo (BID) (Núñez y Oneto, 2012), que se refiere al gobierno corporativo en lo que respecta a la emisión de instrumentos de deuda. Se construyó con base en estándares internacionales, incluye los principios de gobierno corporativo de la Organización de Cooperación y Desarrollo Económicos (OCDE, 2016), y agrupa 9 categorías y 19 estándares (véase el cuadro 2) ${ }^{8}$.

6 En Núñez y Oneto (2015) se afirma que, al aplicar un indicador elaborado para medir la calidad del gobierno corporativo de 22 empresas de cinco países de la región, los resultados obtenidos mostraron que en la mayoría de las empresas evaluadas no existía un comité corporativo dedicado a monitorear el nivel de riesgo de forma permanente e integral. En general, en la región, y particularmente en el MILA y en el Brasil, tanto el comité de riesgos como el comité de auditoría son las áreas en que más es necesario mejorar los esquemas de gobierno corporativo.

7 En el análisis de riesgo se privilegia la información sobre el diferencial de los bonos y se entiende el riesgo como la diferencia entre el rendimiento del bono en cuestión y el de un bono cupón cero de igual plazo del Departamento del Tesoro de los Estados Unidos. En el caso de la muestra de empresas que se conformó para el período 2005-2015, dicho diferencial alcanza alrededor del $4,30 \%$.

8 El indicador fue construido por Núñez y Oneto (2012) sobre la base de estándares internacionales relativos a los gobiernos corporativos, como los principios de gobierno corporativo de la OCDE (OCDE, 2016) y de la CAF (2013), así como de estándares nacionales, como The UK Corporate Governance Code (FRC, 2011) y The Combined Code of Corporate Governance (FRC, 2008). El indicador se construyó con el objetivo de contar con una herramienta cualitativa (medible) que reflejara el nivel de riesgo y de control interno específicamente relacionado con los procesos de emisión de títulos de deuda de una empresa. Esto se logra en tres etapas: i) identificación, a nivel agregado, de las principales actividades de los procesos de emisión de deuda en las que el gobierno corporativo tiene responsabilidad directa, entre ellas, determinar las necesidades de financiamiento, seleccionar y aprobar los intermediarios financieros que estarán a cargo de la emisión, determinar los riesgos de la emisión y hacer un seguimiento de su evolución, autorizar la emisión con base en la información sobre el uso de los fondos recaudados y las implicancias del apalancamiento, así como estructurar los sistemas de control interno que informen oportunamente sobre la efectividad del control del riesgo y el desempeño de los directivos; ii) definición de estándares de gobierno corporativo que tienen un impacto en los procesos de emisión de deuda, y iii) definición de los criterios que determinan la importancia de los estándares específicos. Véanse más detalles sobre el indicador en Núñez y Oneto (2012). 


\section{Cuadro 2}

Indicador de evaluación de las normas de gobierno corporativo respecto de las emisiones de deuda internacional

\begin{tabular}{|c|c|c|c|c|}
\hline Categoría & & Estándar & $\begin{array}{l}\text { Ponderación } \\
\text { (en porcentajes) }\end{array}$ & $\begin{array}{l}\text { Número de } \\
\text { preguntas }\end{array}$ \\
\hline \multirow[t]{2}{*}{$\begin{array}{l}\text { Papel del Consejo de } \\
\text { Administración }\end{array}$} & 1 & $\begin{array}{l}\text { El Consejo de Administración deberá establecer los mecanismos } \\
\text { que le aseguren información confiable sobre todas las actividades } \\
\text { de inversión en activos financieros y no financieros, y las } \\
\text { actividades de financiamiento que realice la empresa. }\end{array}$ & 15,52 & 1 \\
\hline & 2 & $\begin{array}{l}\text { El Consejo de Administración solo podrá delegar } \\
\text { responsabilidades y funciones en los comités corporativos } \\
\text { presididos por un consejero externo independiente. }\end{array}$ & 5,84 & 1 \\
\hline $\begin{array}{l}\text { Estructura del Consejo } \\
\text { de Administración }\end{array}$ & 3 & $\begin{array}{l}\text { El tamaño adecuado del Consejo de Administración será } \\
\text { aquel que le permita tomar decisiones en forma ágil. }\end{array}$ & 0,94 & 2 \\
\hline \multirow[t]{2}{*}{$\begin{array}{l}\text { Papel del presidente del } \\
\text { Consejo de Administración }\end{array}$} & 4 & $\begin{array}{l}\text { El presidente del Consejo de Administración deberá establecer } \\
\text { los mecanismos que conduzcan a la selección de consejeros } \\
\text { externos con base en el valor que pueden aportar. }\end{array}$ & 3,98 & 1 \\
\hline & 5 & $\begin{array}{l}\text { El presidente del Consejo de Administración deberá } \\
\text { ser un consejero externo independiente. }\end{array}$ & 1,88 & 1 \\
\hline $\begin{array}{l}\text { Papel y selección de los } \\
\text { consejeros ejecutivos o } \\
\text { internos y de los consejeros } \\
\text { no ejecutivos o externos }\end{array}$ & 6 & $\begin{array}{l}\text { Los consejeros deberán mantenerse actualizados sobre } \\
\text { las necesidades de la empresa y de sus empleados. }\end{array}$ & 7,79 & 2 \\
\hline $\begin{array}{l}\text { Consejeros externos } \\
0 \text { independientes }\end{array}$ & 7 & $\begin{array}{l}\text { Los consejeros externos deberán transparentar ante el Consejo de } \\
\text { Administración cualquier conflicto de interés relacionado con la empresa. }\end{array}$ & 5,84 & 1 \\
\hline \multirow[t]{2}{*}{ Consejeros internos } & 8 & $\begin{array}{l}\text { Los consejeros internos deberán firmar certificados que } \\
\text { los responsabilicen legal y penalmente de la información } \\
\text { que generen y difundan, así como de la omisión de } \\
\text { divulgar información al Consejo de Administración. }\end{array}$ & 7,77 & 2 \\
\hline & 9 & $\begin{array}{l}\text { El director de Auditoría Interna deberá ser un miembro } \\
\text { del Consejo de Administración y depender directamente } \\
\text { de este o de alguno de sus comités corporativos. }\end{array}$ & & \\
\hline \multirow[t]{4}{*}{ Comité de Auditoría } & 10 & $\begin{array}{l}\text { El Comité de Auditoría deberá ser presidido por un consejero } \\
\text { externo independiente, con experiencia en control interno. } \\
\text { El auditor externo deberá ser contratado por el Comité } \\
\text { de Auditoría y depender directamente de él. }\end{array}$ & 19,40 & 2 \\
\hline & 11 & $\begin{array}{l}\text { El Comité de Auditoría deberá aprobar los programas de auditoría } \\
\text { y dar seguimiento a la atención de las observaciones de esta. }\end{array}$ & & \\
\hline & 12 & $\begin{array}{l}\text { El Comité de Auditoría deberá aprobar el diseño y el } \\
\text { funcionamiento del sistema de control interno. }\end{array}$ & & \\
\hline & 13 & $\begin{array}{l}\text { El Comité de Auditoría será responsable de asegurar que se } \\
\text { tenga un sistema de información efectivo, especialmente } \\
\text { sobre los aspectos financieros, el control de riesgos y el } \\
\text { desempeño de la empresa y de sus directivos. }\end{array}$ & & \\
\hline \multirow[t]{2}{*}{$\begin{array}{l}\text { Comité de Financiamiento } \\
\text { Corporativo }\end{array}$} & 14 & $\begin{array}{l}\text { El Comité de Financiamiento Corporativo deberá ser presidido por un } \\
\text { consejero externo independiente con experiencia en la materia. } \\
\text { El Comité de Financiamiento Corporativo deberá } \\
\text { dictaminar sobre las necesidades de financiamiento y los } \\
\text { mecanismos propuestos por la dirección general. }\end{array}$ & 15,52 & 4 \\
\hline & 15 & $\begin{array}{l}\text { El Comité de Financiamiento Corporativo deberá aprobar la } \\
\text { selección y contratación de los intermediarios financieros que } \\
\text { requiera la empresa para la colocación de los títulos financieros. }\end{array}$ & & \\
\hline \multirow[t]{4}{*}{ Comité de Riesgos } & 16 & $\begin{array}{l}\text { El Comité de Riesgos deberá ser presidido por un consejero externo } \\
\text { independiente, con experiencia en administración integral de riesgos. }\end{array}$ & 15,52 & 3 \\
\hline & 17 & $\begin{array}{l}\text { El Comité de Riesgos deberá dictaminar sobre los informes de los riesgos } \\
\text { financieros y de crédito elaborados por el área de riesgos de la empresa. }\end{array}$ & & \\
\hline & 18 & $\begin{array}{l}\text { El Comité de Riesgos deberá presentar informes periódicos } \\
\text { a la Dirección General y al Consejo de Administración } \\
\text { sobre la efectividad de la estrategia de inversiones. }\end{array}$ & & \\
\hline & 19 & $\begin{array}{l}\text { El Comité de Riesgos deberá aprobar el plan de tratamiento } \\
\text { (mitigación, contención o traspaso) de los riesgos no financieros } \\
\text { que presente la Dirección General de la empresa. }\end{array}$ & & \\
\hline Total & & & 100 & \\
\hline
\end{tabular}

Fuente: Elaboración propia, sobre la base de G. Núñez y A. Oneto (coords.), "Gobernanza corporativa en el Brasil, Colombia y México: la determinación del riesgo en la emisión de instrumentos de deuda corporativa", Documentos de Proyectos (LC/W.468), Santiago, Comisión Económica para América Latina y el Caribe (CEPAL), 2012. 
Los tres primeros niveles (el papel del Consejo de Administración, su estructura y el papel de su presidente) forman la estructura general del gobierno corporativo. Luego hay tres comités corporativos especializados que proporcionan información al Consejo de Administración respecto a temas más específicos sobre la emisión de deuda. El indicador está basado en preguntas que dan como resultado una respuesta binaria: si la empresa cumple, se adopta el valor $1 \mathrm{y}$, si no cumple, el valor 0 . El indicador es normalizado adquiriendo valores entre 0 y 10 . Aunque el indicador se creó para evaluar directamente el desempeño del gobierno de 22 empresas emisoras de deuda del Brasil y los cuatro países del MILA, su utilidad puede extenderse a la evaluación de las leyes y los códigos de buenas prácticas vigentes que rigen dicha actividad. Para esta investigación se adaptaron los estándares y las preguntas originales, así como sus ponderaciones. Se mantuvieron casi todas las categorías, con excepción del Comité de Inversiones Financieras, y se adaptaron las preguntas relacionadas con cada estándar, tomando en consideración solo la normativa y los códigos de buenas prácticas correspondientes y combinando los niveles obligatorio (marco normativo) y voluntario (códigos de buenas prácticas de gobernanza). La ponderación por categoría se mantuvo igual, y la del comité excluido se distribuyó entre las demás. En la matriz de estándares que figura en el cuadro 2, que permite evaluar la normativa sobre gobierno corporativo, la transparencia es un aspecto transversal que abarca las distintas categorías del indicador: está presente en la mayoría de los estándares y justifica la necesidad de aplicar el indicador a cualquier tipo de emisión de deuda (internacional o nacional) y no solo a las emisoras públicas o las que están registradas en los mercados de valores.

Las normas revisadas vigentes se insertan en el ámbito del mercado de capitales y los códigos de buenas prácticas de gobierno corporativo en su mayoría forman parte de la información que las bolsas de valores solicitan periódicamente. Se utiliza el indicador para evaluar estas normas en relación con cada uno de los países seleccionados y se obtiene un solo valor por país y por año. La obligatoriedad de suministrar información sobre la actividad de la empresa a través de su gobierno corporativo en muchos casos es discrecional y está basada en el principio de "cumpla o explique". Lo anterior, aunado al número limitado de empresas sujetas a las normas de los gobiernos corporativos, puede neutralizar el posible efecto reductor de las asimetrías de información.

Un tema que vale la pena mencionar y que ha estado presente en las modificaciones del marco de normas de los gobiernos corporativos es el énfasis que se le da a la presencia de un mayor número de consejeros independientes en los directorios. En muchos de los marcos se han observado mejoras sustantivas al respecto. Esto es especialmente importante en los esquemas de propiedad concentrada, donde el controlador suele poseer más del $50 \%$ de la propiedad. Al evaluar la normativa y los códigos mediante el indicador se obtuvieron los valores que se resumen en el cuadro 3. Los resultados de la medición del nivel general de las normas sobre gobierno corporativo en los cuatro países del MILA y en el Brasil muestran que el desempeño de las entidades regulatorias de los cinco países es bastante similar, y que, en los últimos años, se ha hecho un esfuerzo por mejorar y hacer confluir los marcos normativos.

Cuadro 3

América Latina (5 países): resultados del indicador de los gobiernos corporativos, 2005-2015

\begin{tabular}{llllllllllll}
\hline País & $\mathbf{2 0 0 5}$ & $\mathbf{2 0 0 6}$ & $\mathbf{2 0 0 7}$ & $\mathbf{2 0 0 8}$ & $\mathbf{2 0 0 9}$ & $\mathbf{2 0 1 0}$ & $\mathbf{2 0 1 1}$ & $\mathbf{2 0 1 2}$ & $\mathbf{2 0 1 3}$ & $\mathbf{2 0 1 4}$ & $\mathbf{2 0 1 5}$ \\
\hline Brasil & 4,27 & 4,27 & 5,06 & 5,06 & 5,06 & 5,06 & 5,06 & 6,14 & 6,14 & 6,14 & 6,14 \\
\hline Chile & 2,56 & 2,56 & 2,56 & 2,56 & 2,56 & 3,06 & 3,06 & 3,58 & 6,84 & 6,84 & 7,25 \\
\hline Colombia & 0,93 & 2,99 & 2,99 & 5,12 & 5,12 & 5,12 & 5,12 & 5,12 & 5,12 & 5,12 & 7,66 \\
\hline México & 4,48 & 6,19 & 6,19 & 6,19 & 6,19 & 6,19 & 6,45 & 6,45 & 6,45 & 6,71 & 7,12 \\
\hline Perú & 4,39 & 4,39 & 4,39 & 4,39 & 4,39 & 4,39 & 4,39 & 4,39 & 4,39 & 7,76 & 7,76 \\
\hline
\end{tabular}

Fuente: Elaboración propia, sobre la base de información oficial de los países. 
El país con el menor índice en 2015 fue el Brasil, lo que se explica por el modo en que se analiza y maneja el riesgo, y por una disposición referida a que un tercio del Consejo de Administración puede estar integrado por ejecutivos de la empresa (un potencial conflicto de interés). En las normas de ese país se considera obligatoria la existencia de un Consejo de Administración, pero también la de un Consejo Fiscal. La normativa de gobierno corporativo del Perú, a su vez, fue la que exhibió el nivel más alto en relación con los otros países en dicho año. Por otra parte, entre 2005 y 2015, Colombia fue el país en que más se mejoró esta normativa. De los cinco países, Chile es el único que no cuenta con un código de buenas prácticas.

Respecto al nivel de cobertura de las categorías del indicador en cada una de las normativas, la de Chile es en la que mejor se aborda el papel del Consejo de Administración en la empresa (estándar 1). Con la reforma de la Ley de Sociedades Anónimas (18.046) de 2009, Chile alcanzó el mayor puntaje en esta categoría. En cuanto a la estructura del Consejo de Administración (estándar 2), las normativas de Colombia y México se destacan por requerir que al menos el 25\% de los consejeros sean independientes ${ }^{9}$. Respecto al papel del presidente del Consejo de Administración (estándar 3), ninguna de las normativas analizadas se distingue de las otras. Sin embargo, en Chile, Colombia y el Perú se prohíbe de forma explícita que, directa o indirectamente, el gerente general sea el mismo que ejerza la presidencia del Consejo de Administración. En relación con los comités de auditoría (estándar 4) y de riesgo (estándar 5), se observan evoluciones distintas. De 2004 a 2015 hubo un esfuerzo en todas las legislaciones, salvo en la del Perú, por hacer obligatoria la existencia de un comité relacionado con cuestiones de auditoría, lo que no ha sido posible observar en el ámbito del análisis exclusivo del riesgo y el financiamiento corporativo.

En general, las mayores mejoras del marco legal de los gobiernos corporativos de los cuatro países del MILA y del Brasil se realizaron entre 2013 y 2014. En el caso de los países del MILA probablemente haya influido la necesidad de armonizar las leyes entre ellos para alcanzar el objetivo de dicha iniciativa: integrar las bolsas de valores de los cuatro países. Por otra parte, los cambios en algunos casos coincidieron con escándalos empresariales en algunos países (por ejemplo, el caso de la empresa La Polar en Chile) y con reformas sectoriales en otros (las reformas de México). Las calificaciones más bajas se obtienen en cuanto a la información sobre los comités corporativos y sus responsabilidades, en especial sobre los temas de riesgo y auditoría. El financiamiento en la emisión de deuda es una información que se considera relevante, sin embargo, son pocas las empresas que cuentan con comités corporativos especializados en el tema. En las normativas no existen disposiciones legales específicas en las que se dé un tratamiento diferenciado a las empresas que emiten deuda y son propiedad del Estado. Los resultados de Núñez y Oneto (2015) mostraron que la estructura de los gobiernos corporativos de las empresas estatales a las que se les aplicó el indicador incluía alguna instancia especializada en los temas de financiamiento e inversiones financieras, lo que en general no se encontró en la mayoría de las estructuras de las empresas privadas consideradas en la muestra ${ }^{10}$.

\footnotetext{
9 Bhagat y Bolton (2008) muestran que la independencia del directorio se correlaciona de forma negativa con el desempeño operacional de la empresa. Esto es especialmente relevante, dado el protagonismo alcanzado por los directorios independientes dentro de los requisitos de las empresas que cotizan en bolsa.

${ }^{10}$ En la muestra del informe de Núñez y Oneto (2015) se incluyen empresas propiedad del Estado, que en general mostraron un desempeño mejor de su gobierno corporativo que las empresas privadas representantes de los otros sectores de la economía (financiero, servicios y manufacturero). En este sentido, se destacan las empresas de Chile y el Perú, cuyo desempeño fue mejor fundamentalmente porque cuentan con al menos tres de los comités corporativos que se consideran en el indicador, lo que contrasta con la realidad de las demás empresas de la muestra. Hay una mayor rigidez de las reglas a nivel de los mercados internos (de acciones y de deuda local), que se traslada a los mercados internacionales de deuda.
} 


\section{Análisis econométrico}

La base de datos que se utilizó en el presente análisis está conformada por emisiones de deuda internacional registradas en la plataforma de Bloomberg durante el período 2005-2015. En total se trata de 5.173 emisiones de bonos internacionales de 323 empresas que pertenecen a un universo de 2.130 empresas de los cuatro países del MILA y el Brasil. Esta muestra se seleccionó a partir de las empresas que contaban con información de activos y deuda en al menos uno de los años del período analizado. Utilizamos el enfoque de "residencia del emisor" para clasificar el mercado de bonos corporativos ${ }^{11}$. Se excluyeron las emisiones nacionales (domestic y domestic MTM) y las colocaciones privadas (private placements), lo que asegura que las categorías no excluidas corresponden solamente a emisiones internacionales realizadas por casas matrices y subsidiarias. El vencimiento promedio de los bonos era de 10 años, con una mediana de 8. Además, las emisiones se realizaron en 15 monedas distintas, si bien el $80 \%$ de ellas fueron en dólares estadounidenses.

La decisión de emitir un bono se puede representar como una variable binaria ficticia (dummy), Bond $_{i t}$, que toma dos valores: sí $=1$ con probabilidad $p_{i}$, no $=0$ con probabilidad $\left(1-p_{i}\right)$. Es decir:

$$
\operatorname{Prob}\left(\text { Bond }_{i t}=1 \mid x, \beta\right)=p_{i}=F(x, \beta)
$$

Dadas las características de nuestra base de datos, se eligió en principio un modelo probit para datos de panel en el cual $F(x, \beta)$ es la función de distribución acumulada de la distribución normal estándar $\int_{-\infty}^{x \beta} \frac{1}{\sqrt{2 \pi}} e^{-\frac{z^{2}}{2}} d z$. El modelo por estimar es el siguiente:

$$
\text { Bond }_{i t}=x_{i t}^{\prime} \beta+\varepsilon_{i t}
$$

La estimación de los parámetros se puede llevar a cabo encontrando los que maximizan el logaritmo de la función de verosimilitud conjunta:

$$
\ln L(\beta)=\sum_{i=1}^{n} \sum_{t=1}^{T}\left\{\left(\text { Bond }_{i t}\right) \ln F\left(x_{i}, \beta\right)+\left(1-\text { Bond }_{i t}\right) \ln \left(1-F\left(x_{i}, \beta\right)\right)\right\}
$$

Mizen y Tsoukas (2012) mencionan cinco razones contables para evaluar la salud financiera de la empresa mediante datos de los estados financieros, y tres de ellas son consideradas en el presente análisis. La primera es la razón de apalancamiento rezagada un período, $L e v R_{i t-1}$ (total de deuda/total de activos). Si el valor de esta razón es alto, o bien los inversionistas pueden pensar que la empresa no será capaz de cumplir con sus obligaciones y no desear comprar el bono (relación negativa), o bien pueden considerar que la empresa es sujeto de crédito, lo que incrementa la probabilidad de demanda del bono (relación positiva). El cuadrado de la variable anterior, $L e v R_{i t-1}^{2}$, se incluye para verificar si hay un nivel máximo (óptimo) de deuda relativa a los activos después del cual pueda cambiar la dirección que sigue la relación entre la variable dependiente y el apalancamiento de la empresa (véase Margaritis y Psillaki, 2010). Para capturar el efecto de la rentabilidad se incluye la variable ProfR $_{i t-1}$, definida como los beneficios antes de intereses e impuestos (EBIT)/los activos totales: unos EBIT menores se relacionan con la probabilidad de que la empresa busque financiamiento externo.

\footnotetext{
${ }^{11}$ En el enfoque de residencia del emisor (párrafo 7.61, BPI/BCE/FMI, 2015), los títulos de deuda emitidos por un residente del país donde se originó el bono se clasifican como emisiones nacionales, independientemente de la moneda de emisión. Todas las otras emisiones se clasifican como emisiones internacionales.
} 
En nuestro modelo, a estas variables sumamos otras. Una de ellas es Size_it, que corresponde al tamaño de la empresa. Se considera que, a mayor tamaño, la probabilidad de que se haga una emisión es mayor. Otra variable es $C E M B I$, que es un índice de referencia de los bonos corporativos denominados en dólares emitidos por entidades de los mercados emergentes. Otra es el índice de gobierno corporativo CorpGov_jt, que mide el desempeño de acuerdo con la normativa y se espera que se relacione positivamente con la probabilidad de que la empresa se financie a través del mercado de renta fija. Dicha expectativa se debe a que la reputación del emisor derivada de un buen desempeño en términos de dicha normativa puede influir en la probabilidad de colocar los bonos con éxito. En el modelo se incluyen asimismo las variables Deriv y $\operatorname{Var} T C$, que miden, respectivamente, el tamaño del mercado de derivados según información del Banco de Pagos Internacionales, y las variaciones del tipo de cambio respecto del dólar en el país de la empresa emisora. También se incluyeron variables ficticias para tomar en cuenta el efecto que pudiera tener el año en cuestión, así como la importancia del sector de actividad de la empresa. Con base en lo anterior, la especificación del modelo a estimar es:

$$
\begin{aligned}
& P\left(\operatorname{Bond}_{i, j, t}=1\right)=F\left(\operatorname{CorpGov}_{i, j, t}+\operatorname{LevR}_{i, j, t-1}+\operatorname{LevR}_{i, j, t-1}^{2}+\operatorname{ProfR}_{i, j, t-1}+\operatorname{Size}_{i, j, t-1}+\right. \\
& \left.\operatorname{CEMBI}_{i, j, t-1}+\operatorname{Deriv}_{i, j, t-1}+\operatorname{VarTC}_{, t-1}+\sum D Y j_{, t}+\sum D S_{z}\right)
\end{aligned}
$$

Donde $i$ representa el emisor individual, $j$ indica el país del emisor, $z$ es el sector donde opera la empresa y $t$ es el período de tiempo.

Para estimar la ecuación anterior, utilizamos dos métodos: un modelo probit con efectos aleatorios para datos de panel, y un modelo pooled probit. Analizamos un total de 6.457 observaciones. Para corregir el problema potencial de endogeneidad por simultaneidad, incluimos todas las variables explicativas con un rezago. De esta forma, se reduce la probabilidad de que las variables independientes estén correlacionadas con el residuo contemporáneo. En el cuadro 4 se muestran los resultados de las estimaciones.

\section{Cuadro 4}

Países del Mercado Integrado Latinoamericano (MILA)

\begin{tabular}{|c|c|c|c|c|}
\hline Variables & $\begin{array}{c}(1) \\
\text { Probit RE } 3\end{array}$ & $\begin{array}{c}\text { (2) } \\
\text { Efectos marg. }\end{array}$ & $\begin{array}{c}\text { (3) } \\
\text { Pooled Probit } 3 \\
\end{array}$ & $\begin{array}{c}\text { (4) } \\
\text { Efectos marg. }\end{array}$ \\
\hline Índice de gobierno corporativo (L.CorpGov) & $\begin{array}{l}0,144^{\star \star \star} \\
(0,0509)\end{array}$ & $\begin{array}{r}0,00252^{\star *} \\
(0,00102)\end{array}$ & $\begin{array}{l}0,120^{\star \star \star} \\
(0,0342)\end{array}$ & $\begin{array}{r}0,00247^{\star \star} \\
(0,00105)\end{array}$ \\
\hline $\begin{array}{l}\text { Razón de apalancamiento rezagada } \\
\text { un período (L.LevR) }\end{array}$ & $\begin{array}{r}4,693^{\star \star \star} \\
(0,830)\end{array}$ & $\begin{array}{r}0,0819^{\star \star \star} \\
(0,0222)\end{array}$ & $\begin{array}{r}4,665^{\star \star \star} \\
(0,719)\end{array}$ & $\begin{array}{r}0,0960^{\star \star \star} \\
(0,0153)\end{array}$ \\
\hline Cuadrado de L.LevR (L.LevR²) & $\begin{array}{r}-4,575^{\star \star *} \\
(1,096)\end{array}$ & $\begin{array}{r}-0,0799^{* * *} \\
(0,0251)\end{array}$ & $\begin{array}{r}-4,576^{\star \star \star} \\
(0,971)\end{array}$ & $\begin{array}{r}-0,0942^{\star \star \star} \\
(0,00787)\end{array}$ \\
\hline $\begin{array}{l}\text { Beneficios antes de intereses e } \\
\text { impuestos/activos totales (L.ProfR) }\end{array}$ & $\begin{array}{l}-1,32 \mathrm{e}-08 \\
(1,02 \mathrm{e}-05)\end{array}$ & $\begin{array}{l}-2,31 e-10 \\
(1,78 \mathrm{e}-07)\end{array}$ & $\begin{array}{r}-1,14 \mathrm{e}-08^{\star \star \star} \\
(7,47 \mathrm{e}-10)\end{array}$ & $\begin{array}{r}-2,34 \mathrm{e}-10^{\star \star \star} \\
(7,27 \mathrm{e}-11)\end{array}$ \\
\hline Tamaño de la empresa (L.Size) & $\begin{array}{l}0,200^{\star \star *} \\
(0,0254)\end{array}$ & $\begin{array}{l}0,00350^{\star \star *} \\
(0,000816)\end{array}$ & $\begin{array}{l}0,144^{\star \star \star} \\
(0,0168)\end{array}$ & $\begin{array}{l}0,00296^{\star \star \star} \\
(0,000912)\end{array}$ \\
\hline $\begin{array}{l}\text { Índice de bonos corporativos emitidos por } \\
\text { entidades de los mercados emergentes (L.CEMBI) }\end{array}$ & $\begin{array}{r}-0,0137^{\star * *} \\
(0,00457)\end{array}$ & $\begin{array}{r}-0,000240 * * \\
(9,34 \mathrm{e}-05)\end{array}$ & $\begin{array}{r}-0,00958^{\star \star \star} \\
(0,00324)\end{array}$ & $\begin{array}{r}-0,000197^{\star \star} \\
(8,56 e-05)\end{array}$ \\
\hline Tamaño del mercado de derivados (L.Deriv) & $\begin{array}{r}2,92 \mathrm{e}-05^{\star \star \star} \\
(1,09 \mathrm{e}-05)\end{array}$ & $\begin{array}{r}5,10 \mathrm{e}-07^{\star *} \\
(2,22 \mathrm{e}-07)\end{array}$ & $\begin{array}{r}2,75 \mathrm{e}-05^{\star \star *} \\
(7,06 \mathrm{e}-06)\end{array}$ & $\begin{array}{r}5,66 \mathrm{e}-07^{\star \star \star} \\
(2,09 \mathrm{e}-07)\end{array}$ \\
\hline $\begin{array}{l}\text { Variación del tipo de cambio } \\
\text { respecto del dólar (L.VarTC) }\end{array}$ & $\begin{array}{l}-0,0117^{\star *} \\
(0,00590)\end{array}$ & $\begin{array}{r}-0,000204^{*} \\
(0,000109)\end{array}$ & $\begin{array}{r}-0,00763^{\star *} \\
(0,00383)\end{array}$ & $\begin{array}{r}-0,000157^{*} \\
(9,53 e-05)\end{array}$ \\
\hline Materiales básicos & $\begin{array}{r}0,761^{* \star \star} \\
(0,248)\end{array}$ & $\begin{array}{l}0,0133^{\star \star \star} \\
(0,00512)\end{array}$ & $\begin{array}{r}0,659^{\star \star \star} \\
(0,182)\end{array}$ & $\begin{array}{l}0,0136^{\star \star \star} \\
(0,00515)\end{array}$ \\
\hline Comunicaciones & $\begin{array}{l}0,685^{\star \star} \\
(0,287)\end{array}$ & $\begin{array}{l}0,0120^{\star *} \\
(0,00561)\end{array}$ & $\begin{array}{r}0,623^{\star \star \star} \\
(0,208)\end{array}$ & $\begin{array}{c}0,0128^{\star \star} \\
(0,00569)\end{array}$ \\
\hline Energía & $\begin{array}{r}1,342^{* \star \star} \\
(0,334)\end{array}$ & $\begin{array}{l}0,0234^{\star \star \star} \\
(0,00746)\end{array}$ & $\begin{array}{r}1,252^{\star \star \star} \\
(0,269)\end{array}$ & $\begin{array}{l}0,0258^{\star \star \star} \\
(0,00941)\end{array}$ \\
\hline Variable ficticia de 2008 (D2008) & $\begin{array}{r}-1,151^{* \star \star} \\
(0,253)\end{array}$ & $\begin{array}{r}-0,0201^{\star \star \star} \\
(0,00575)\end{array}$ & $\begin{array}{r}-0,831^{\star \star \star} \\
(0,174)\end{array}$ & $\begin{array}{r}-0,0171^{\star * \star} \\
(0,00620)\end{array}$ \\
\hline
\end{tabular}
y Brasil: modelos probit estimados, 2005-2015 
Cuadro 4 (conclusión)

\begin{tabular}{|c|c|c|c|c|}
\hline Variables & $\begin{array}{c}(1) \\
\text { ProbitRE } 3\end{array}$ & $\begin{array}{c}\text { (2) } \\
\text { Efectos marg. }\end{array}$ & $\begin{array}{c}\text { (3) } \\
\text { Pooled Probit3 }\end{array}$ & $\begin{array}{c}\text { (4) } \\
\text { Efectos marg. }\end{array}$ \\
\hline Variable ficticia de 2012 (D2012) & $\begin{array}{l}0,365^{\star *} \\
(0,155)\end{array}$ & $\begin{array}{l}0,00637^{\star *} \\
(0,00294)\end{array}$ & $\begin{array}{r}0,186^{\star} \\
(0,0975)\end{array}$ & $\begin{array}{r}0,00383 \\
(0,00237)\end{array}$ \\
\hline Variable ficticia de 2013 (D2013) & $\begin{array}{r}0,511^{\star \star \star} \\
(0,164)\end{array}$ & $\begin{array}{r}0,00892^{\star \star \star} \\
(0,00330)\end{array}$ & $\begin{array}{r}0,301^{\star \star \star} \\
(0,103)\end{array}$ & $\begin{array}{l}0,00619^{\star \star} \\
(0,00280)\end{array}$ \\
\hline Variable ficticia de 2014 (D2014) & $\begin{array}{r}0,461^{\star \star \star} \\
(0,161) \\
\end{array}$ & $\begin{array}{l}0,00805^{\star \star} \\
(0,00314)\end{array}$ & $\begin{array}{l}0,239^{\star \star} \\
(0,105)\end{array}$ & $\begin{array}{l}0,00493^{*} \\
(0,00265)\end{array}$ \\
\hline Constante & $\begin{array}{r}-4,833^{\star \star \star *} \\
(0,614)\end{array}$ & & 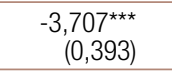 & \\
\hline Observaciones & 8,817 & 8,817 & 8,817 & 8,817 \\
\hline Número de id & 1,514 & & & \\
\hline Valor $p$ de Wald & $<0$ & & $<0$ & \\
\hline Criterio de información de Akaike (AIC) & 1965 & & 2297 & \\
\hline Criterio de información bayesiano (BIC) & 2163 & & 2489 & \\
\hline Pseudo R2 & & & 0,221 & \\
\hline Porcentajes de aciertos estimados (PCP) & & & 96,22 & \\
\hline
\end{tabular}

Fuente: Elaboración propia.

Nota: Error estándar entre paréntesis: ${ }^{\star * \star} p<0,01,{ }^{\star *} p<0,05 y * p<0,1$.

Id: identificaciones de unidades de panel únicas (ticker Bloomberg).

Como puede observarse, el coeficiente asociado con el indicador de gobierno corporativo no solo resultó altamente significativo, sino que además tiene signo positivo, lo cual sugiere que la probabilidad de que la emisión de bonos internacionales se lleve a cabo con éxito se ve incrementada en la medida en que se satisfacen estándares más altos de gobierno corporativo. Por otra parte, el coeficiente relacionado con la medida de apalancamiento muestra un signo positivo, además de ser también muy significativo. Esto indica que las empresas que han tenido experiencia en la emisión previa de deuda podrían estar beneficiándose de ella, lo que les permitiría incrementar sus probabilidades ulteriores de emitir con éxito en el mercado internacional de deuda corporativa. Es interesante observar que el término estimado en relación con el cuadrado de la medida de apalancamiento también es muy significativo de acuerdo con los niveles convencionales. Sin embargo, aunque su magnitud numérica es similar a la del coeficiente de apalancamiento, el cuadrado tiene signo negativo en ambos modelos, lo que sugiere que puede existir un nivel óptimo de endeudamiento más allá del cual se obtienen efectos decrecientes sobre la posibilidad de tener éxito al emitir nueva deuda. El tamaño de la empresa también muestra una relación muy significativa y positiva, lo que apoya la hipótesis de que el tamaño de la empresa puede contribuir al éxito en la emisión de bonos en los mercados internacionales.

De acuerdo con las estimaciones, la afiliación a los sectores de la energía, las materias básicas y las comunicaciones tuvo, en ese orden, efectos positivos de gran significancia en la probabilidad de tener éxito al emitir bonos internacionales. La variable ficticia correspondiente a 2008 tuvo una gran significancia y signo negativo, en tanto que los coeficientes de 2012, 2013 y 2014 resultaron significativos y tuvieron signo positivo.

\section{Conclusión}

La importancia del mercado de los bonos corporativos en el financiamiento de las empresas de América Latina ha aumentado sostenidamente desde 2009. Esta influencia creciente puede atribuirse en parte a la coyuntura favorable (bajas tasas de interés) que prevaleció en la economía mundial derivada de la respuesta de los gobiernos ante la crisis financiera de 2008, así como a la política de expansión cuantitativa de la Reserva Federal de los Estados Unidos, que influyó notablemente en la tasa de rendimiento del mercado internacional de bonos. Sin embargo, este contexto también representa una fuente de riesgo, dado que al cambiar las condiciones favorables 
mundiales (en particular, la posición de la autoridad monetaria estadounidense), se podría revertir el proceso de financiamiento de las empresas latinoamericanas y ello podría tener efectos relevantes en la estabilidad de las economías de la región.

En este artículo presentamos los resultados que se obtuvieron al analizar la importancia del cumplimiento de los principios y las buenas prácticas de gobierno corporativo en el Brasil y en los cuatro países latinoamericanos que forman parte del Mercado Integrado Latinoamericano (MILA). En particular, se utilizó el indicador de la CEPAL, la CAF y el BID relativo a las emisiones de deuda y, mediante un análisis econométrico basado en un modelo probit para datos de panel y un modelo pooled probit, se encontró que un mayor nivel de cumplimiento de los principios y estándares de buenas prácticas de gobierno corporativo puede contribuir significativamente a incrementar las probabilidades de que una empresa tenga éxito al emitir bonos internacionales. Las pruebas presentadas en este artículo resaltan la importancia que puede tener un buen gobierno corporativo en cuanto garante de la transparencia, al divulgar de forma eficiente información relevante en tiempo y cantidad adecuados. Naturalmente, estos resultados también pueden entenderse como indicio de que una regulación del marco corporativo que minimice los conflictos de interés y reduzca las asimetrías de información puede ayudar a que las empresas tengan un mejor acceso a los mercados internacionales de bonos a fin de obtener el financiamiento necesario para desarrollar sus actividades productivas.

\section{Bibliografía}

Bhagat, S. y B. Bolton (2008), "Corporate governance and firm performance", Journal of Corporate Finance, vol. 14, No 3, Amsterdam, Elsevier.

BPI (Banco de Pagos Internacionales) (2016a), "Debt securities statistics" [en línea] https://www.bis.org/ statistics/secstats.htm?m=6\%7C33\%7C615.

(2016b), "OTS derivatives statistics at end-December 2015", mayo [en línea] https://www.bis.org/publ/ otc_hy1605.htm.

2015), "Triennial Central Bank Survey of Foreign Exchange and Derivatives Market Activity in 2013" [en línea] http://www.bis.org/publ/rpfx13.htm.

BPI/BCE/FMI (Banco de Pagos Internacionales/Banco Central Europeo/Fondo Monetario Internacional) (2015), Handbook on Securities Statistics, Washington, D.C., Fondo Monetario Internacional (FMI).

CAF (Corporación Andina de Fomento) (2013), Lineamientos para un código latinoamericano de gobierno corporativo [en línea] http://publicaciones.caf.com/media/25389/ineamientos_codigo_latinoamericano.pdf.

CBOE (Chicago Board Options Exchange) [en línea] https://www.cboe.com/micro/vix/vixwhite.pdf.

Çelik, S., G. Demirtas y M. Isaksson (2015), "Corporate bonds, bondholders and corporate governance", OECD Corporate Governance Working Papers, N 16, París, OECD Publishing.

Financial Times (2016), 14 de julio.

FMI (Fondo Monetario Internacional) (2015), "Coordinated Portfolio Investment Survey (CPIS)" [en línea] http://data.imf.org/?sk=B981B4E3-4E58-467E-9B90-9DE0C3367363.

FRC (Financial Reporting Council) (2011), Guidance on Board Effectiveness, Londres, marzo. (2008), Combined Code of Corporate Governance, Londres, junio.

Gozzi, J. C. y otros (2015), "How firms use corporate bond markets under financial globalization", Journal of Banking \& Finance, vol. 58, Amsterdam, Elsevier.

G20/OCDE (Grupo de los 20/Organización de Cooperación y Desarrollo Económicos) (2015), G20/OECD Principles of Corporate Governance. OECD Report to G20 Finance Ministers and Central Bank Governors [en línea] https://www.oecd.org/daf/ca/Corporate-Governance-Principles-ENG.pdf.

J. P. Morgan (2016), Fixed Income Index Product Guide, Londres, enero.

Margaritis, D. y M. Psillaki (2010), "Capital structure, equity ownership and firm performance", Journal of Banking \& Finance, vol. 34, N 3, Amsterdam, Elsevier.

MILA (Mercado Integrado Latinoamericano) [en línea] mercadomila.com. 
Mizen, P. y S. Tsoukas (2012), "The response of the external finance premium in Asian corporate bond markets to financial characteristics, financial constraints and two financial crises", Journal of Banking \& Finance, vol. 36, № 11, Amsterdam, Elsevier.

Núñez, G. y A. Oneto (coords.) (2015), "Corporate governance in Brazil, Chile, Colombia, Mexico and Peru: the determinants of risk in corporate debt issuance", Documentos de Proyectos, № 654 (LCM.654), Santiago, Comisión Económica para América Latina y el Caribe (CEPAL) [en línea] https://repositorio. cepal.org/handle/11362/37721.

(2014), "La gobernanza corporativa y la emisión de deuda corporativa en Chile y el Perú: el papel de los inversionistas institucionales, las agencias calificadoras de riesgo y la banca de inversión", Documentos de Proyectos, № 624 (LC/W.624), Santiago, Comisión Económica para América Latina y el Caribe (CEPAL) [en línea] https://repositorio.cepal.org/handle/11362/37387.

_ (coords.) (2012), "Gobernanza corporativa en el Brasil, Colombia y México: la determinación del riesgo en la emisión de instrumentos de deuda corporativa", Documentos de Proyectos (LC/W.468), Santiago, Comisión Económica para América Latina y el Caribe (CEPAL).

OCDE (Organización de Cooperación y Desarrollo Económicos) (2016), Principios de gobierno corporativo de la OCDE y del G20, París, OECD Publishing.

Pérez Caldentey, E. (2017), "Quantitative easing (QE), changes in global liquidity and financial instability", Documento de Trabajo, № 1701, Post Keynesian Economics Study Group [en línea] www.postkeynesian. net/downloads/working-papers/PKWP1701_Oo3Adk2.pdf.

Rodrigues Bastos, F., H. Kamil y B. Sutton (2015), "Corporate financing trends and balance sheet risks in Latin America", IMF Working Papers, № 15/10, Washington, D.C., Fondo Monetario Internacional (FMI).

Rogoff, K. (2015), "Was quantitative easing the best way to boost the U.S. economy?", The Boston Globe, 1 de marzo [en línea] https://www.bostonglobe.com/business/2015/03/01/was-best-way-boosteconomy/2A3u8T6IWLzPpPRmssRd4K/story.html.

Tromben, C. y otros (2015), "Se busca director", América Economía, № 107, mayo-junio.

WFE (World Federation of Exchanges) [en línea] https://www.world-exchanges.org/home/index.php/statistics. 